\title{
Characteristic identification of oil dampers for railway vehicles using Neural Networks
}

\author{
R. Koganei, K. Sasaki \& N. Watanabe \\ Railway Technical Research Institute, Tokyo, Japan
}

\begin{abstract}
We developed a characteristic identification system for devices of railway vehicles which is an essential component in a system of 'Virtual Running Test Environment' based on Hardware In the Loop Simulation (HILS) technology, as developed by Railway Technical Research Institute (RTRI) to replace running tests with bench tests. This report describes the outline and the effect of the characteristic identification system for oil dampers using a Neural Network $(\mathrm{NN})$, which estimates input-output relation of the target in 6 degree-of-freedom using multi-axis damper test equipment.
\end{abstract}

Keywords: railway vehicle, oil damper, Neural Network, hardware in the loop simulation, characteristic identification.

\section{Introduction}

Running tests are indispensable to develop the railway vehicle. In Japan, however, a great amount of cost and time are required for the tests. In addition, most of the running tests take place on service lines since there is no test truck available for exclusive use in Japan. Accordingly, a number of test and the test condition are restricted.

It is likely to lead to shortening and the quality improvement of the development process if it comes to be able to reproduce a real railway vehicle motion in detail by some bench examinations.

Then, we work the construction of 'Virtual Running Test Environment' based on Hardware In the Loop Simulation (HILS) technology (Fig. 1), which replaces running tests with bench tests. It is necessary to mount the greater part of the railway vehicle components on the simulation model as software, because it is impractical to mount all of the railway vehicle components as hardware in 
composing this HILS system. However, it is difficult to obtain a high accuracy model for the simulation using a past simulation procedure, because there are some components which have strong nonlinear characteristic.

For example, one of the components is oil damper for railway vehicle. The oil dampers are installed between trucks and car-body for the railway vehicle, which are component that suppress vibration. This component contributes improvement of ride comfort and running stability. It is problem to determine characteristic of the damper properly for the railway vehicle development.

On the other hands, rubber bushes of elasticity material are assembled in both ends of the damper. It can allow for movements other than main axis. However, the specification test method of the dampers in JIS (Japanese Industrial Standards) contains no influence of the rigidity of the installation part.

Evaluating the characteristics of damper including the rubber bush is important to understand that the elasticity material exerts influence on the characteristic of damper and that the generated force of the damper exerts on the characteristic of the vehicle movement when the railway vehicle is running.

Then, we developed the damper test equipment (Fig. 2) that is able to excite the damper by three dimensions and to obtain the generated force of the damper as like as the real running. In addition, we formulated a characteristic identification technique for automatically of the damper. The technique obtains a high-precision model of the damper including the nonlinear characteristic from the experimental data.

In this report, we introduce the characteristic identification system using a NN (Neural Network) for the dampers, which estimates input-output relation of the target in 6-DOF (Degree of Freedom) using multi-axis damper test equipment. In addition, we show the identification results applying this technique.

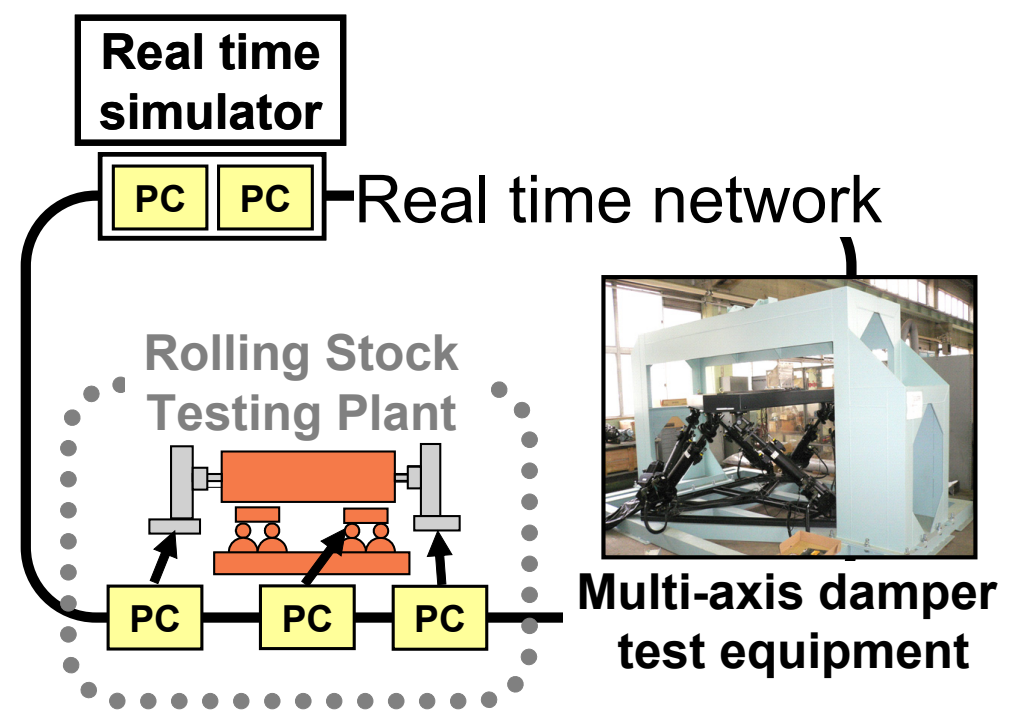

Figure 1: $\quad$ HILS system. 


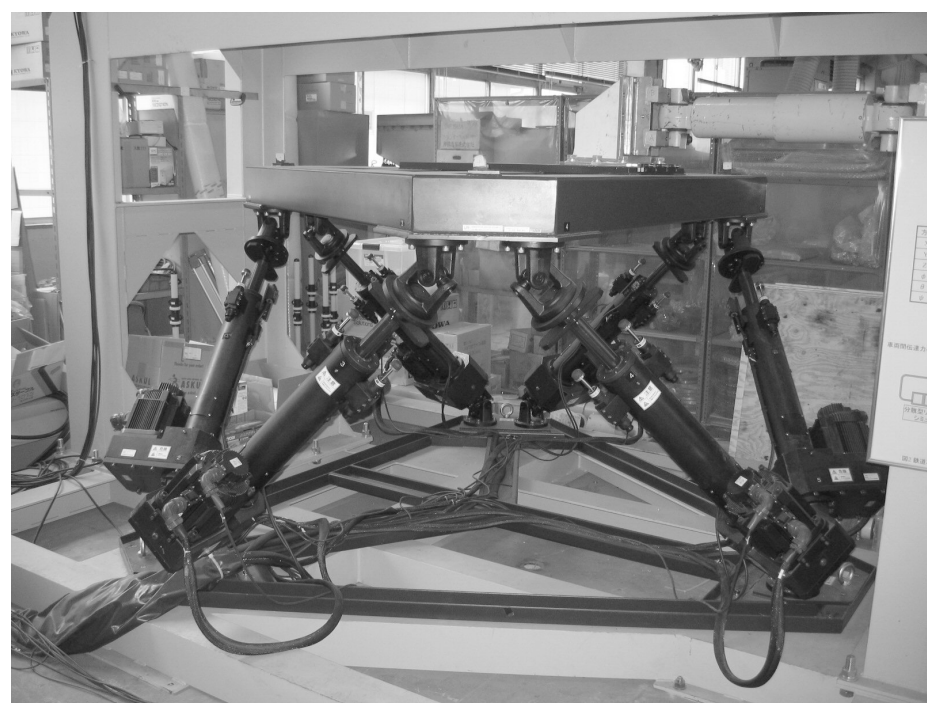

Figure 2: Multi-axis damper test equipment.

\section{Training using Neural Network}

\subsection{Neural Network}

There are some indispensable points in a predicted method that we need. First, the method is possible to correspond to an object with a strong nonlinear characteristic. Second, the method is possible to identify without entering the model structure. Third, the method is possible to make the precision model automatically. Therefore, we selected a method as based on the Neural Network $(\mathrm{NN})$. The $\mathrm{NN}$ is one technique for making it self-optimizing to obtain an output signal from an arbitrary input signal by training the input-output relation.

\subsection{How to prepare training data}

It is necessary to obtain an input-output data for training as called training data. In the obtained $\mathrm{NN}$ model, if an arbitrary input data includes to the training data, the NN model outputs a highly accurate result. On the other hands, an accuracy of the predicted result decreases remarkably, when the data does not include to the training data. Then, the stroke and velocity of the training data generated that they have enough range to include the real running one. Figure 3 shows the distribution range chart of the piston stroke and the velocity of the damper obtained by running test comparing with the training data. The multi-axis damper test equipment drives the target damper foregoing the training data, and the damper generates the damping force. After that, the relation between the damping force, displacement and the velocity is identifiable using the NN. 


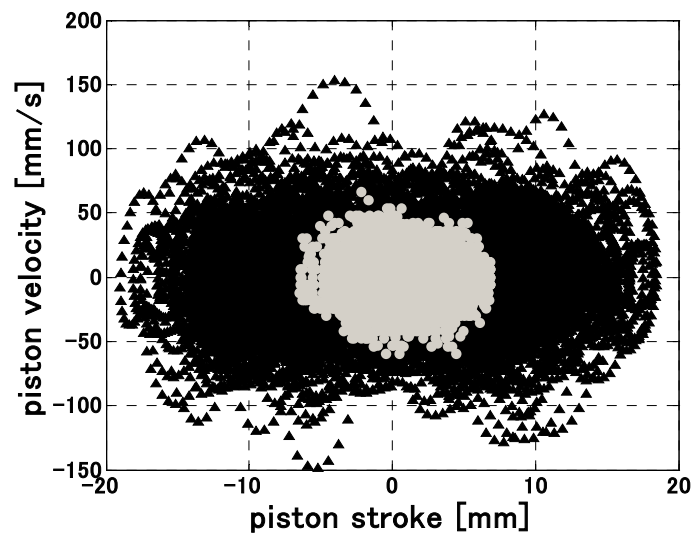

Figure 3: The distribution chart of the training data (black points: training data, grey points: measured data).

\subsection{Model combined NN and simple dynamics}

There are two types of network structures of the NN model. One is "Recurrent type" with a feedback from the output to the input. Another is "Feed forward type" without feedback. The former method is capable of indicating the inputoutput relation using a few order, though, there is a possibility to diverge by some momentary anomaly data. The latter method is less affected by anomalous data, though it needs to increase the number of input if the target has a complex characteristic. Therefore, we selected the latter method considering the stability as the HILS system.

A proposed NN model is as shown in Fig.4 (a). This model has two inputs and one output, and has a nonlinear part and a linear part. In the case of some type of dampers, the rubber bush makes down coherence between the input and the output. This fact signifies that it is difficult to obtain a highly accurate model by the feed forward type NN, without some supplemental ways. Thus, we proposed a method of improving accuracy using intermediate information with high coherence. The new proposed model is NN model including a simple dynamic model (piecewise linear model) of identification target as apparent in Fig.4 (b). Figure 5 shows the piecewise linear model. The relation with the damping force and piston velocity is as shown in equation (1) and (2).

$$
\begin{aligned}
& \qquad \begin{array}{l}
f=\left(x-x_{1}\right) k \\
\text { when } \quad|f|<f_{r},
\end{array} \\
& \qquad \begin{aligned}
& f=c \dot{x}_{1} \\
& \text { when } \quad|f| \geq f_{r}, \\
& f=\operatorname{sgn}\left(\dot{x}_{1}\right)\left(1-c_{2} / c_{1}\right) f_{r}+c_{2} \dot{x}_{1}
\end{aligned}
\end{aligned}
$$




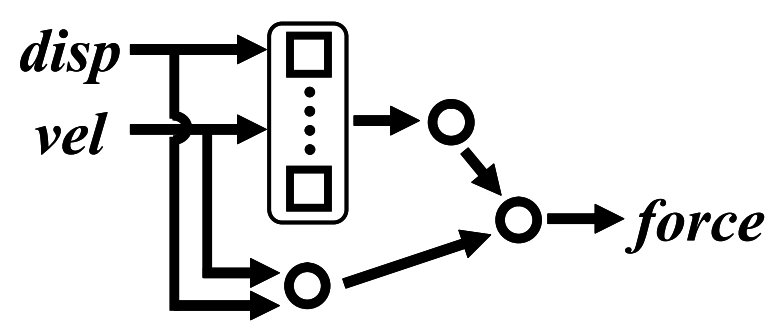

(a) $\mathrm{NN}$ model

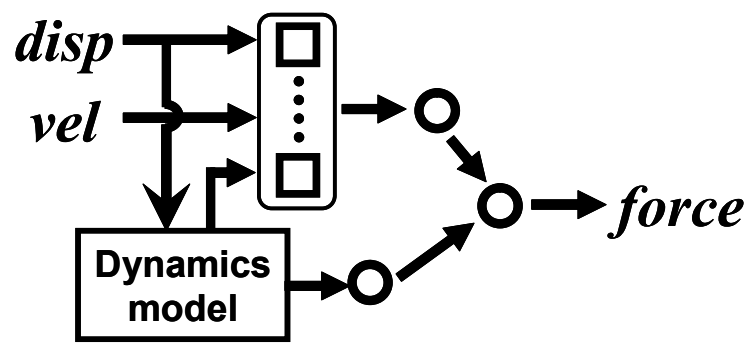

$\square$ : nonlinear neuron

$\circ$ : linear neuron

(b) NN Model Combined Dynamics Model

Figure 4: Model for characteristic identification.

In this faction, the optimize parameters that minimizes the difference between the approximate damping force and the measurement damping force are determined by Newton method. The initial parameters for optimization are set as the nominal values of the corresponding damper. The nominal values and the optimized values are as shown in Table 1 .

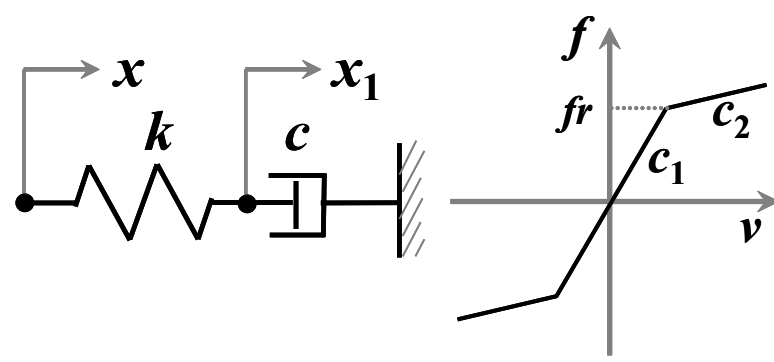

$x$ : displacement

$x_{1}$ : displacement of hidden states

$k$ : constant of spring

$c$ : damping coefficient $f r$ : damping force

$v$ : piston velocity

$c_{1}$ : damping coefficient

$c_{2}$ : damping coefficient

Figure 5: $\quad$ Piecewise linear model. 
Table 1: $\quad$ Comparison of nominal parameters with optimized parameters.

\begin{tabular}{|l|c|c|}
\hline & nominal value & optimal value \\
\hline Damping coefficient $: c 1[\mathrm{Ns} / \mathrm{m}]$ & $2.452 \times 10^{6}$ & $2.305 \times 10^{6}$ \\
\hline Damping coefficient $: c 2[\mathrm{Ns} / \mathrm{m}]$ & $1.798 \times 10^{5}$ & $1.684 \times 10^{5}$ \\
\hline Constant of spring $: k[\mathrm{~N} / \mathrm{m}]$ & $6.15 \times 10^{6}$ & $2.457 \times 10^{6}$ \\
\hline Damping force on changing point $: f r[\mathrm{~N}]$ & $8.826 \times 10^{3}$ & $7.603 \times 10^{3}$ \\
\hline
\end{tabular}

\section{Characteristic identification of damper on main axis}

\subsection{Excitation waveform}

As previously mentioned, the training data range should have as larger than the actual stroke and piston velocity of running railway vehicle. Thus, the excitation data is generated as greater amplitude and the same spectrum as the damper stroke of the running test. The damper test equipment excites the target damper with the foregoing the excitation data to obtain the training data.

\subsection{Result of characteristic identification}

The validity of the obtained model was verified by using no correlation data with the training data. The results of comparison the measurement value with the predicted value are as shown in Fig. 6. This figure shows the lateral and anti yawing dampers. The predicted result by the ARX model that is the typical linear identification technique is described in parallel.

Since the ARX model is a linear identification method, the precision of estimate depends on the nonlinearity of the target. In the lateral damper, which has comparatively weak nonlinearity, the predicted wave shape resembles to the measured one except the amplitude of several peak points. In contrast, as the anti yawing damper has strong nonlinearity, the predicted wave shape is greatly different to the measured shape.

On the other hands, NN shows excellent predict results for both dampers, and they are almost corresponding to the actual measurement values. MSE (Mean Square of Error) between the measured force and the predicted force using NN has decreased compared to the ARX model by $33 \%$ (for lateral damper) and $76 \%$ (for anti yawing damper) (Fig. 6).

\section{Characteristic identification for actual running condition}

\subsection{Relations between multi-axis input and multi-axis output}

The multi-axis damper test equipment has the ability of 6-DOF excitation that appears in actual running, to clarify the influence of six axes inputs (displacement) and six outputs (force, torque). For example, it is confirmed that the excitation except main axis generates the damping force in the direction of the main axis in anti yawing dampers. 

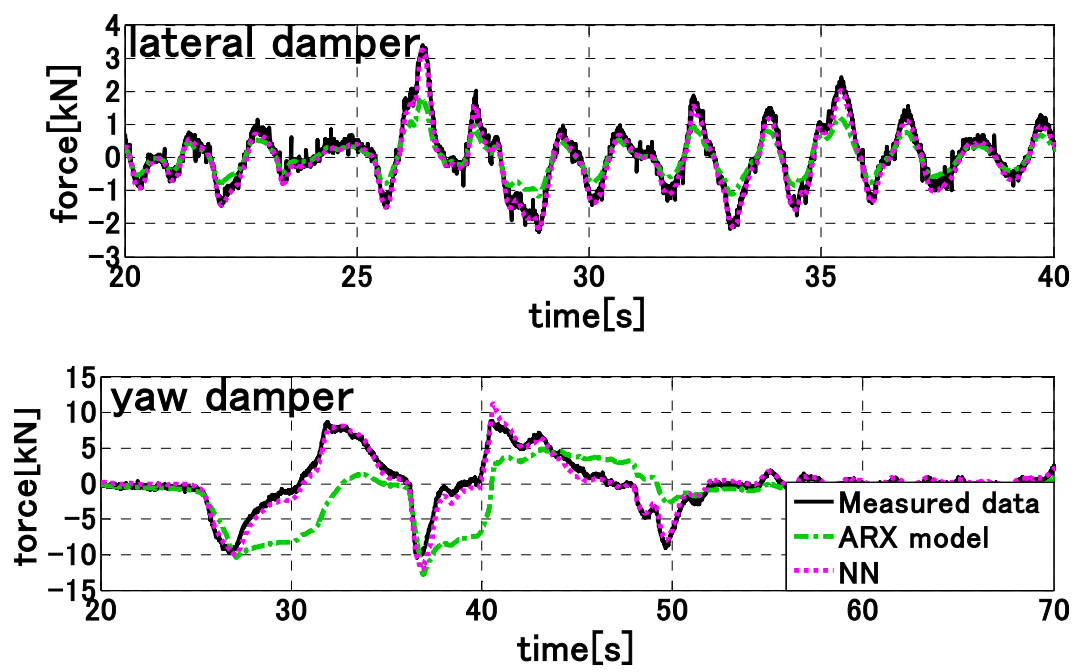

Figure 6: Comparison of the measured force with predicted force using the ARX model and NN.

It shows that the identification model of multi degree of freedom is necessary to consider not only the direction of the main axis but also the influence of two or more axes to reproduce damper behaviour accurately of the damper in an actual running.
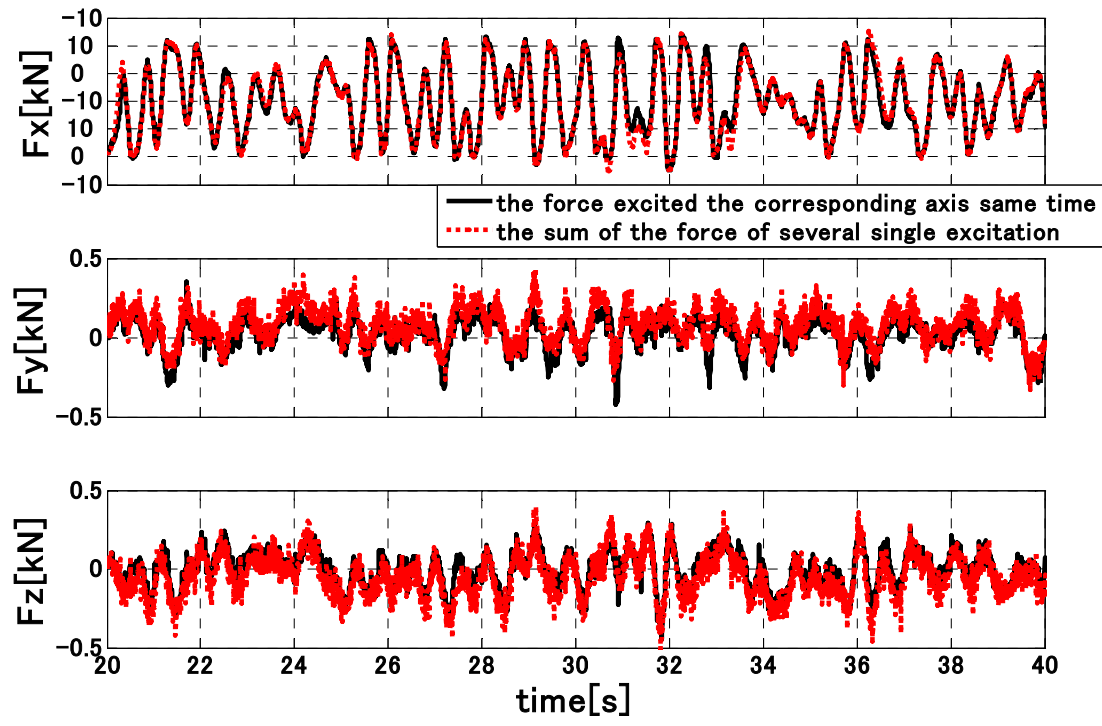

Figure 7: Comparison of the sum of the force of several single excitation with the force which excited the corresponding axis same time. 


\subsection{Excitation waveform}

The sum of the force of several single excitations agrees approximately to the force, which by the composite excited a corresponding axis (Fig. 7). To identify characteristic, the exciting test requires only once for each axis. Training data is synthesized with the single axis excitation data to meet where required.

\subsection{Result of characteristic identification}

The multi-axis NN model is configured as a set of single output models, which are corresponding to each axis outputs. Each sub models use input signals, which have strong correlation to the output. Figure 8 is an example of the sub model. This model predicts main axis force of anti yawing damper from the displacement of three axes. By this structure, this model is added to estimate the main axis force by the orthogonal displacement to the main axis.

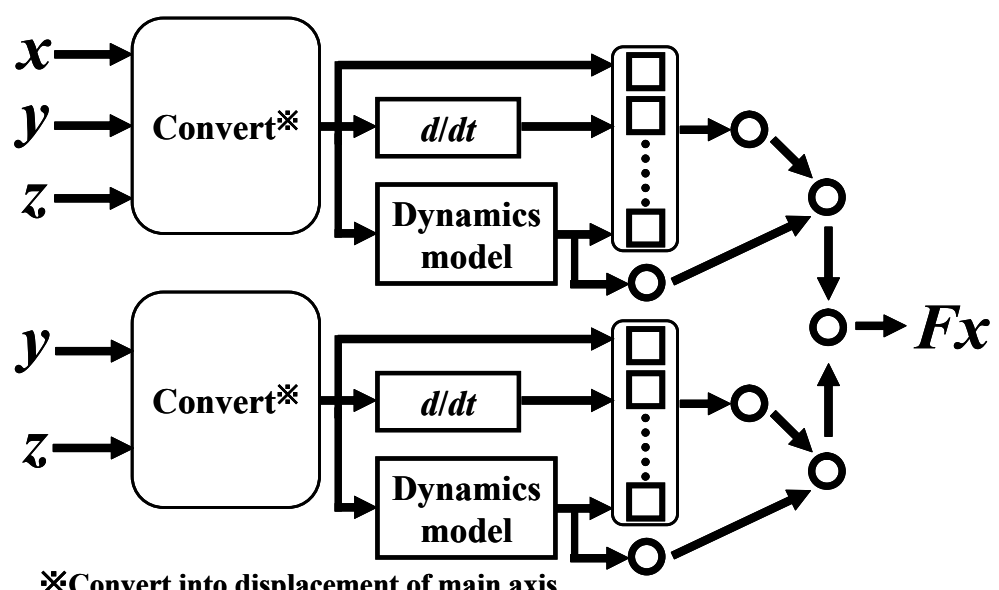

Figure 8: $\quad$ NN model in 6-DOF ( for anti yawing damper.

Figure 9 shows the comparison of the predicted forces and torques by the NN model with the experimental data. The figure shows that all the predicted outputs are consistent with the corresponding experimental value.

\section{Conclusion}

In this report, we propose a highly accurate characteristic identification system using a NN (Neural Network) for oil dampers, which estimates the input-output relation of the target in 6-DOF (Degree of Freedom) using a multi-axis damper test equipment. The obtained knowledge is as shown below.

It has shown how to prepare the 6-DOF data for identification using 6-DOF oil damper test equipment, which reproduces substantial railway vehicle motions in detail. 

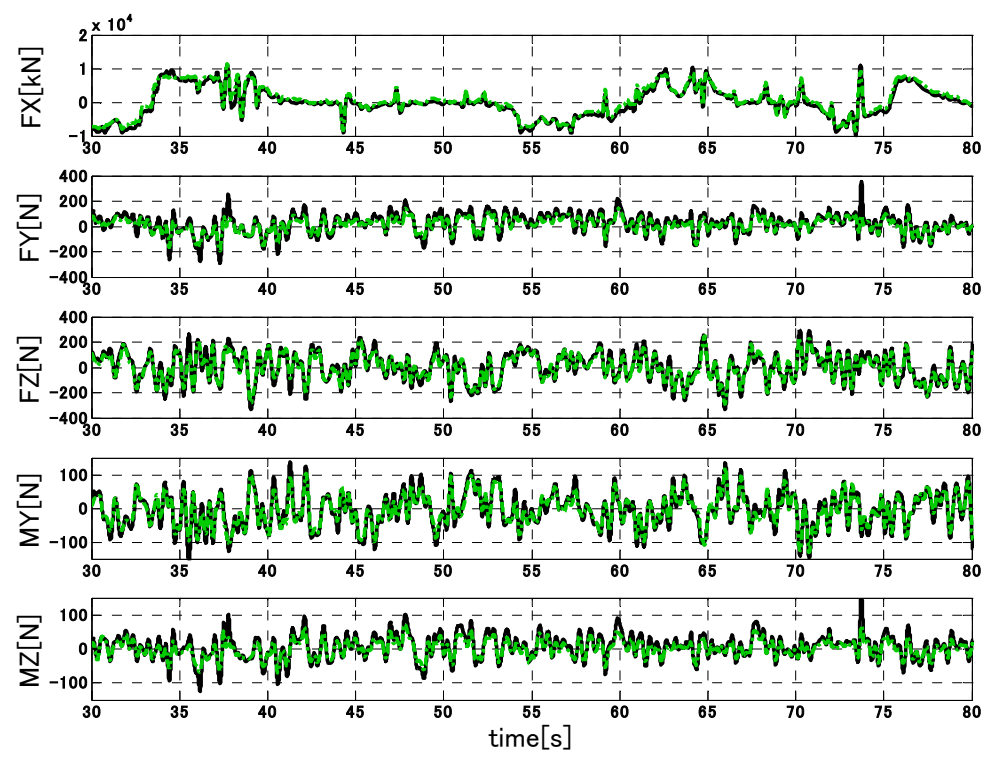

Figure 9: $\quad$ Comparative Measured Force with predicted force using NN in 6DOF (anti yawing damper) (Black line: measured force, Gray line: predicted force using $\mathrm{NN}$ ).

In the case of the system including hidden states, the coherence between the input (displacement) and output (force, torque) becomes low. Therefore we proposed a new type NN model including a simple dynamic model (piecewise linear model).

The learning data range is selected as larger than the actual stroke and piston velocity of actual running railway vehicle.

As the result of identification of main axis characteristic, the predicted force using the ARX model was different from the measured force. Contrarily, the measured force and the predicted force using NN were almost consistent. MSE (Mean Square of Error) between the measured force and the predicted force using $\mathrm{NN}$ has decreased by $33 \%$ (for lateral damper) or $76 \%$ (for anti yawing damper) compared with the ARX model.

The model of main axis has been enhanced to 6-DOF model. The 6-DOF high-predicted model was obtained.

\section{References}

[1] M. Nagai, A. Moran, Y. Tamura and S. Koizumi, Identification and control of nonlinear active pneumatic suspension for railway vehicles, using neural network, Control Eng. Practice, Vol. 5, No. 8, pp. 1137-1144, 1997

[2] Y. Umehara, N. Watanabe, M. Asahina and R. Koganei, A Study of Virtual Running Test of Railway Vehicle, The 8th World Congress on Railway Research, 2008 